\title{
Incompatibility between Propericiazine Oral Solution and Tea-Based Drink
}

\author{
Hirohito Ikeda, ${ }^{* a}$ Erika Tsuji, ${ }^{a}$ Tomonori Matsubara, ${ }^{a}$ Miho Yukawa, ${ }^{a}$ Masao Fujisawa, ${ }^{b}$ \\ Eiji Yukawa, ${ }^{c}$ and Hatsumi $\mathrm{Aki}^{a}$ \\ ${ }^{a}$ Faculty of Pharmaceutical Sciences, Fukuoka University; 8-19-1 Nanakuma, Jonan-ku, Fukuoka 814-0180, Japan: \\ ${ }^{b}$ Department of Biotechnological Sciences, Kinki University; 930 Nishimitani, Kinokawa, Wakayama 649-6493, \\ Japan: and ${ }^{c}$ Faculty of Pharmaceutical Sciences, Daiichi University of Pharmacy; 22-1 Tamagawa-cho, Minami-ku, \\ Fukuoka 815-8511, Japan. Received February 6, 2012; accepted July 1, 2012
}

\begin{abstract}
Here, we studied the incompatibility between an oral solution of propericiazine (PCZ), an antipsychotic drug, and various commercially available bottled tea-based drinks. When $0.5 \mathrm{~mL}$ of the PCZ oral solution $(10 \mathrm{mg} / \mathrm{mL})$ was mixed with $16.5 \mathrm{~mL}$ of a tea-based drink (such as green tea, oolong tea, and black tea), the residual PCZ content declined to approximately $50 \%$ in some mixed solutions. After mixing with other tea-based drinks, the residual PCZ content declined to approximately $30 \%$, while in others, it changed very little. The residual PCZ content declined immediately after mixing with tea-based drinks, but the rate remained almost unchanged for the next $24 \mathrm{~h}$. Furthermore, the pH of the mixture increased to $4.5-5.1$ after the oral solution of PCZ (original pH 3.8) was diluted with various tea-based drinks. Afterwards, the pH did not change for $24 \mathrm{~h}$. The mixture became cloudy immediately after diluting PCZ oral solution with tea-based drinks, and the insoluble substance gradually precipitated. In order to elucidate factors responsible for the decline in the content of PCZ, a (-)-epigallocatechin gallate solution, which is a main ingredient of green tea polyphenol, was mixed with the PCZ oral solution. After mixing, the residual PCZ content declined to approximately 60-75\%. On the other hand, the content of PCZ did not decline when a (-)-epigallocatechin solution was mixed with the $\mathrm{PCZ}$ oral solution. The results from this study demonstrated that $\mathrm{PCZ}$ content was reduced after dilution in tea-based drinks because of the interaction between $\mathrm{PCZ}$ and polyphenol with a galloyl group in tea-based drinks.
\end{abstract}

Key words incompatibility; propericiazine; tea-based drink; polyphenol; galloyl group

In July 2011, the Ministry of Health, Labour, and Welfare (MHLW) of Japan added psychiatric disorders to their medical care plan, which had already contained 4 major diseases (cancer, cerebral stroke, heart disease, and diabetes). According to the 2008 Patient Survey conducted by the MHLW, the number of patients with psychiatric disorder was approximately 3.23 million, which exceeded the number of patients with cancer or diabetes. The number is expected to further increase in the future. The most common psychiatric disorder is depression, followed by schizophrenia and anxiety disorders. The majority of patients with psychiatric disorders undergo drug therapy. Schizophrenia is the most common psychiatric disorder in Japan with a lifetime prevalence of approximately $1 \%$. Schizophrenia is a chronic disease with periods of remission and relapse. There are 3 main symptoms: positive symptoms (hallucination and auditory hallucination), negative symptoms (lack of motivation and apathy), and cognitive impairment (attention disturbance). Therefore, in order to improve symptoms and prevent relapse, patient compliance with drug therapy is important. However, even if doctors and pharmacists provide patients with appropriate instructions for compliance, schizophrenic patients often have strong preferences and may not follow these instructions. Keith and Kane reported that greater than $90 \%$ of doctors believed that their patients with schizophrenia were non-compliant. This rate of non-compliance was greater than that of patients with hypertension, diabetes, or depression. The report also indicated that patients who discontinued drug administration within 7 to $10 \mathrm{~d}, 1$ year, and 2 years after starting treatment for schizophrenia were $25 \%$,

The authors declare no conflict of interest.
$50 \%$, and $75 \%$, respectively. ${ }^{1)}$ Various factors contributed to the poor adherence and non-compliance with drug administration and dosage. ${ }^{1,2}$ Therefore, family members, doctors, nurses, and pharmacists should closely cooperate with and support patients with schizophrenia in adhering to their drug therapy.

Propericiazine (PCZ) is a typical antipsychotic agent for the treatment of schizophrenia. It should not be administered as an undiluted solution in order to avoid inadvertent use. Accordingly, the directions for use indicate that the patient should make a diluted solution by mixing a single dose of the PCZ oral solution in water, juice, or soup. ${ }^{3)}$ However, these instructions do not specify appropriate types of diluents.

According to information published in 2011 by the Japan Tea Central Association, Japan is the fourth leading consumer of tea-based drinks in the world. As such, some patients with schizophrenia may dilute PCZ with tea-based drinks, even if they are instructed to take the drug with water. Various biologically active substances in tea-based drinks interact with some drugs and affect their pharmacological activity. If a biologically active substance in a tea-based drink reduces the drug's efficacy, it can be significant problems for effective drug therapy.

Here, we report the incompatibility between a PCZ oral solution and various commercially available bottled tea-based drinks.

\section{Experimental}

Materials The PCZ oral solution, Neuleptil ${ }^{\mathbb{B}}$ oral solution $1 \%$ (Shionogi \& Co., Ltd., Osaka, Japan) was used. The bottled tea-based drinks consisted of 3 types of green tea drinks, 
3 types of oolong tea drinks, 3 types of black tea drinks, and 6 types of other tea-based drinks; these drinks were purchased from a supermarket. (-)-Epigallocatechin gallate (EGCg) was purchased from Sigma-Aldrich (St. Louis, MO, U.S.A.). (-)-Epigallocatechin (EGC) and $\mathrm{NaH}_{2} \mathrm{PO}_{4}$ were purchased from Nacalai Tesque, Inc. (Kyoto, Japan). Acetonitrile and methanol were purchased from, Kanto Chemical Co., Inc. (Tokyo, Japan). All were HPLC-grade or guaranteed reagents.

Measurement of pH of Tea-Based Drink The $\mathrm{pH}$ of each tea-based drink used for this study was measured using a $\mathrm{pH}$ meter (M-8s, Horiba, Ltd.).

Change in PCZ Content after Mixing the PCZ Oral Solution with Tea-Based Drink First, $0.50 \mathrm{~mL}$ of the PCZ oral solution and $16.5 \mathrm{~mL}$ of a tea-based drink were mixed. Then, $0.50 \mathrm{~mL}$ of the mixture was collected immediately after mixing and filtered through a membrane (pore size: $0.45 \mu \mathrm{m}$ ). The PCZ content in the filtrate was determined by HPLC.

Changes in PCZ Content over Time after Mixing the PCZ Oral Solution and Tea-Based Drink One of each kind of tea drink from the above-mentioned experiment (green tea, oolong tea, black tea, and other tea drink) was selected. First, $0.50 \mathrm{~mL}$ of the PCZ oral solution was mixed with $16.5 \mathrm{~mL}$ of the selected tea drink and was stirred with a magnetic stirrer. Then, $0.50 \mathrm{~mL}$ of the mixture was collected immediately or after $2 \mathrm{~h}, 4 \mathrm{~h}$, or $24 \mathrm{~h}$. The solution was filtered through a membrane (pore size: $0.45 \mu \mathrm{m}$ ), and the PCZ content in the filtrate was measured by HPLC.

Changes in $\mathbf{p H}$ and Turbidity over Time after Mixing the PCZ Oral Solution with Tea-Based Drink First, $3.0 \mathrm{~mL}$ of the PCZ oral solution was mixed with $100 \mathrm{~mL}$ of a tea-based drink. The $\mathrm{pH}$ was measured using a $\mathrm{pH}$ meter (M-8s, Horiba, Ltd.). The turbidity of the mixture was measured before and immediately after mixing and $2 \mathrm{~h}, 4 \mathrm{~h}$, and $24 \mathrm{~h}$ after mixing.

Changes in PCZ Content after Mixing the PCZ Oral Solution with the Green Tea Polyphenol Solution The green tea polyphenol (EGCg or EGC) solution was prepared using a citrate buffer solution ( $\mathrm{pH}$ 5.2). The concentration of the polyphenol was $0.25,0.50$, and $1.0 \mathrm{mg} / \mathrm{mL}$. Then, $0.50 \mathrm{~mL}$ of the PCZ oral solution was mixed with $16.5 \mathrm{~mL}$ of the polyphenol solution. The $\mathrm{pH}$ was measured before and immediately after mixing. Then, $0.50 \mathrm{~mL}$ of the mixture was collected, filtered through a membrane (pore size: $0.45 \mu \mathrm{m}$ ), and the residual content of the PCZ and the polyphenol in the filtrate were measured by HPLC. As a control, $0.50 \mathrm{~mL}$ of the PCZ oral solution was mixed with $16.5 \mathrm{~mL}$ of the citrate buffer solution ( $\mathrm{pH}$ 5.2) was prepared, then the PCZ content in the filtrate was measured in the same manner.

HPLC Determination of PCZ HPLC measurement of PCZ was performed 3 times for each sample solution. The HPLC apparatus used in this study consisted of a pump (PU-2080 plus, JASCO Corporation) and a UV detector (UV-2075 plus, JASCO Corporation). A $\mathrm{C}_{18}$ reversed phase column $(250 \mathrm{~mm} \times 4.6 \mathrm{~mm}$, particle size $5.0 \mu \mathrm{m}$, Mightysil RP-18, Kanto Chemical Industry Co., Ltd.) was used, and the column temperature was set at $35.0^{\circ} \mathrm{C}$. A mixed solution of $\mathrm{CH}_{3} \mathrm{CN}, \mathrm{CH}_{3} \mathrm{OH}$, and $30 \mathrm{~mm} \mathrm{NaH} \mathrm{PO}_{4}$ solution (volume ratio $3: 2: 5)$ was used as the mobile phase. The flow rate of the mobile phase was set at $0.9 \mathrm{~mL} / \mathrm{min}$, and the UV absorbance of the eluate was measured at $250 \mathrm{~nm}$.

HPLC Determination of the Green Tea Polyphenol
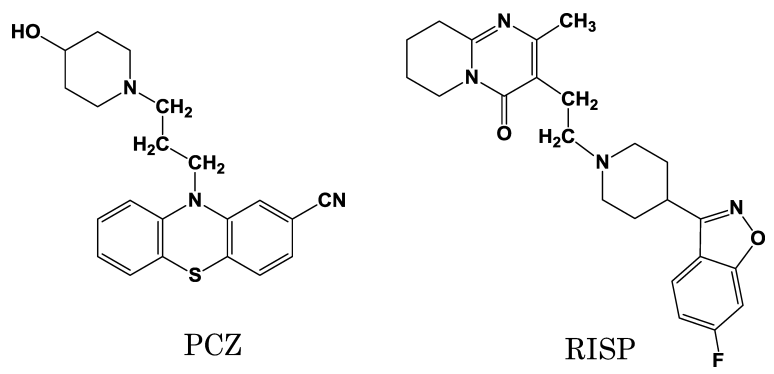

Fig. 1. Chemical Structures of PCZ and RISP

HPLC measurement of the green tea polyphenol was performed 3 times for each sample solution. The HPLC apparatus used in this study consisted of a pump (LC-20AT, Shimadzu Corporation) and a UV detector (SPD-10AV, Shimadzu Corporation). $\mathrm{A}_{18}$ reversed phase column $(250 \mathrm{~mm} \times 4.6 \mathrm{~mm}$, particle size $5.0 \mu \mathrm{m}$, Mightysil RP-18, Kanto Chemical Industry Co., Ltd.) was used, and the column temperature was set at $40.0^{\circ} \mathrm{C}$. A mixed solution of $\mathrm{CH}_{3} \mathrm{OH}$, and $0.5 \mathrm{v} / \mathrm{v} \% \mathrm{H}_{3} \mathrm{PO}_{4}$ solution (volume ratio 18:82) was used as the mobile phase. The flow rate of the mobile phase was set at $1.5 \mathrm{~mL} / \mathrm{min}$, and the UV absorbance of the eluate was measured at $280 \mathrm{~nm}$.

\section{Results and Discussion}

Changes in PCZ Content after Mixing the PCZ Oral Solution with Tea-Based Drink In Japan, PCZ (Fig. 1), haloperidol, risperidone (RISP, Fig. 1), and aripiprazole (APZ) have been administered as liquids and solutions for the treatment of schizophrenia. Among these drugs, the package inserts for RISP and APZ warn that the drug concentration may decline due to an interaction with tea-based drinks. It is thought that this results from the formation of an insoluble complex between the drugs and components of tea-based drinks. However, the full mechanism has yet to be elucidated. A recent study found that the polyphenol, a major ingredient in green tea, is responsible for reduced RISP concentration when the drug is mixed with green tea. Furthermore, ${ }^{1} \mathrm{H}-\mathrm{NMR}$ measurements and molecular modeling using density functional theory identified that hydrogen bonds between the piperidine ring nitrogen of RISP and hydroxyl groups of the galloyl ring of polyphenol were responsible for the formation of an insoluble complex. ${ }^{4,5)}$ As shown in Fig. 1, PCZ has a piperidine ring, similar to RISP, suggesting that it may form insoluble complexes with ingredients of tea-based drinks. In order to test this hypothesis, we studied the change in PCZ content in a mixture of the PCZ oral solution and commercially available bottled tea-based drinks.

The tea-based drinks used in this study were bottled drinks available in supermarkets. Based on the "50 Best-selling tea-based drinks" reported in the Japanese Tea Newspaper, 15 types of tea-based drinks were selected, because these were dominant in the market, easily obtained, and likely preferred by patients. These tea-based drinks included 3 types of green tea drinks (A, B, and C), 3 types of oolong tea drinks (D, E, and $\mathrm{F}$ ), 3 types of black tea drinks (G, H, and $\mathrm{I})$, and 6 types of other tea-based drinks (J, K, L, M, N, and O). J and $\mathrm{K}$ were drinks marketed to relieve and prevent metabolic syndromes; $\mathrm{L}$ was jasmine tea; $\mathrm{M}$ was du zhong tea; $\mathrm{N}$ was barley tea; and $\mathrm{O}$ was a blend of several herbal extracts. The $\mathrm{pH}$ and polyphenol content of each tea-based drink used for this study 
were shown in Table 1. The polyphenol content in Table 1 was provided by the manufacturer of each tea-based drink.

According to the package insert of the PCZ oral solution, ${ }^{3)}$ the maximum daily dose of $\mathrm{PCZ}$ is $60 \mathrm{mg}$. PCZ is indicated for aggressive schizophrenia patients, in whom $150 \mathrm{mg}$ per day is administered in clinical settings. ${ }^{6,7)}$ The PCZ concentration in the oral solution is $10 \mathrm{mg} / \mathrm{mL}$. Therefore, we assumed that patients dilute the daily maximum dose of the PCZ oral solution $(6.0 \mathrm{~mL})$ with 1 glass of tea-based drink $(200 \mathrm{~mL})$. Accordingly, we evaluated the change in PCZ content in a mixture of $0.5 \mathrm{~mL}$ of the $\mathrm{PCZ}$ oral solution and $16.5 \mathrm{~mL}$ of a tea-based drink.

Table 2 shows the residual PCZ content after mixing the PCZ oral solution with each of the 3 types of green tea drinks (A, B, and C), 3 types of oolong tea drinks (D, E, and F), and 3 types of black tea drinks ( $\mathrm{G}, \mathrm{H}$, and I). Table 3 shows the results when the PCZ oral solution was mixed with each of the other 6 types of tea-based drinks (J, K, L, M, N, and O). As shown in Table 2, the PCZ content declined to $45-70 \%$ except when diluted in $\mathrm{H}$.

The polyphenol contents of the green tea $(\mathrm{A}-\mathrm{C})$ were clear as a catechin as shown in Table 1 . The residual PCZ content in the mixture decreased with an increase in the polyphenol content of the green tea. According to the Products A and B were manufactured under the same brand and by the same seller; however, the polyphenol content of Product B was higher than that of Product A. The residual PCZ concentration in B declined by approximately $15 \%$ more than did A. These results suggested that the green tea polyphenol might reduce the residual concentration of PCZ.

$\mathrm{H}$ was a black tea drink containing lemon juice; accordingly, its $\mathrm{pH}$ was approximately 3.8 , which was lower than the other tea-based drinks ( $\mathrm{pH}$ 4.7-6.3) as shown Table 1. The low $\mathrm{pH}$ might have inhibited the reduction in PCZ content. On the other hand, the relationship that the tea-based drink indicating higher $\mathrm{pH}$ before mixing lowers the contents of $\mathrm{PCZ}$ in the mixture was not recognized from Tables 1-3.

As shown in Table 3, the residual PCZ content only slightly declined in $\mathrm{N}$ and $\mathrm{O}$. It is supposed that $\mathrm{N}$ (the barley tea) and $\mathrm{O}$ (the tea blend, consisted of various herbal extracts) did not include the ingredients reducing a content of PCZ. On the other hand, the residual PCZ content in J was significantly reduced. $\mathrm{J}$ contained substantial amounts of polymerized polyphenol derived from oolong tea. It is known that polymerized polyphenol strongly interacts with RISP in solution. ${ }^{8)} \mathrm{We}$ hypothesized that it strongly interacts with PCZ as well.

Changes in PCZ Content over Time after Mixing the PCZ Oral Solution with Tea-Based Drink The highest selling green tea (A), oolong tea (D), and black tea (G) drinks were selected for this experiment. From the other tea drinks, when mixed with the PCZ oral solution, J retained the greatest concentration of PCZ and was selected for this experiment. Figure 2 shows the PCZ content in the mixture immediately after, $2 \mathrm{~h}, 4 \mathrm{~h}$, and $24 \mathrm{~h}$ after mixing $0.50 \mathrm{~mL}$ of the $\mathrm{PCZ}$ oral solution with $16.5 \mathrm{~mL}$ of the selected drink. The residual PCZ content in the mixture with A, D, G, and J declined immediately after mixing but changed very little over the next $24 \mathrm{~h}$.

Changes in pH and Turbidity over Time after Mixing the PCZ Oral Solution with Tea-Based Drink The $\mathrm{pH}$ of the PCZ oral solution used in this experiment was 3.8. The $\mathrm{pH}$ was determined immediately after, $2 \mathrm{~h}, 4 \mathrm{~h}$, and $24 \mathrm{~h}$ after mixing $3.0 \mathrm{~mL}$ of the $\mathrm{PCZ}$ oral solution with $100 \mathrm{~mL}$ of the tea-based drink (A, D, G, or J). Figure 3 shows that the $\mathrm{pH}$ of the mixture increased to 4.5-5.1 immediately after mixing but did not change over the next $24 \mathrm{~h}$.

The turbidity of the mixture was also evaluated. The color and turbidity of the PCZ oral solution before mixing were yellow and clear, respectively. The color of the tea-based drinks used in this experiment was brown to brownish red. The solutions became cloudy immediately after mixing, and the

Table 1. The $\mathrm{pH}$ and Polyphenol Content $(\mathrm{mg} / \mathrm{mL})$ of Tea-Based Drink

\begin{tabular}{|c|c|c|c|c|c|c|c|c|}
\hline Drink & A & B & $\mathrm{C}$ & $\mathrm{D}$ & $\mathrm{E}$ & $\mathrm{F}$ & G & $\mathrm{H}$ \\
\hline $\mathrm{pH}$ & 6.3 & 6.3 & 6.2 & 5.7 & 6.0 & 6.0 & 5.4 & 3.8 \\
\hline Polyphenol & $0.32^{a)}$ & $\left.0.80^{a}\right)$ & $0.34^{a)}$ & $0.10^{b)}$ & - & $0.30^{b)}$ & - & - \\
\hline Drink & I & $\mathrm{J}$ & K & $\mathrm{L}$ & M & $\mathrm{N}$ & $\mathrm{O}$ & \\
\hline $\mathrm{pH}$ & 5.4 & 6.0 & 5.8 & 4.7 & 6.3 & 6.0 & 6.0 & \\
\hline Polyphenol & $0.50^{c)}$ & $0.20^{b)}$ & $0.07^{d)}$ & $0.30^{a)}$ & $0.00^{a)}$ & - & - & \\
\hline
\end{tabular}

- : The manufacturer did not show any data of polyphenol content. $a$ ) as a catechin, $b$ ) as an oolong tea polyphenol, $c$ ) as a tannin, $d$ ) as a guava leaf polyphenol.

Table 2. Residual PCZ Content (\%) in the Mixture of PCZ Oral Solution and Tea-Based Drinks (Green Tea, Oolong Tea and Black Tea)

\begin{tabular}{|c|c|c|c|c|c|c|c|c|c|}
\hline \multirow{2}{*}{ Drink } & \multicolumn{3}{|c|}{ Green tea } & \multicolumn{3}{|c|}{ Oolong tea } & \multicolumn{3}{|c|}{ Black tea } \\
\hline & A & $\mathrm{B}$ & $\mathrm{C}$ & $\mathrm{D}$ & E & $\mathrm{F}$ & G & $\mathrm{H}$ & I \\
\hline PCZ & $68.3 \pm 5.7$ & $53.7 \pm 2.8$ & $57.7 \pm 5.2$ & $51.1 \pm 2.8$ & $47.5 \pm 1.5$ & $45.7 \pm 1.7$ & $53.8 \pm 2.4$ & $83.4 \pm 4.0$ & $55.6 \pm 2.1$ \\
\hline
\end{tabular}

Values $(n=3)$ are means \pm standard deviations of triplicate measurement.

Table 3. Residual PCZ Content (\%) in the Mixture of PCZ Oral Solution and Tea-Based Drinks (Other Tea-Based Drinks)

\begin{tabular}{ccccccc}
\hline \hline Drink & $\mathrm{J}$ & $\mathrm{K}$ & $\mathrm{L}$ & $\mathrm{M}$ & $\mathrm{O}$ & $\mathrm{N}$ \\
\hline $\mathrm{PCZ}$ & $29.1 \pm 2.6$ & $52.2 \pm 4.4$ & $75.5 \pm 6.1$ & $80.7 \pm 2.1$ & $95.8 \pm 3.8$ & $96.9 \pm 4.7$ \\
\hline
\end{tabular}

Values $(n=3)$ are means \pm standard deviations of triplicate measurement. 


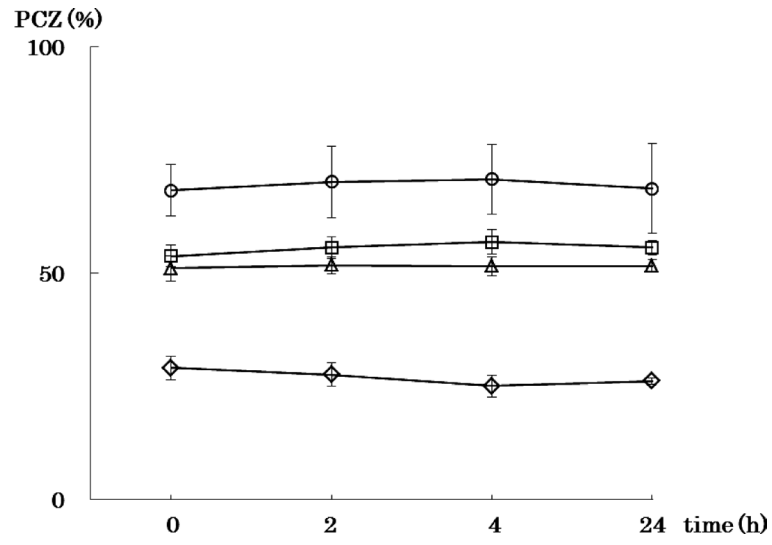

Fig. 2. Time Course of Changes in PCZ Content (\%) over Time after Mixing the PCZ Oral Solution with Tea-Based Drinks (A, D, G and J)

$\mathrm{O}: \mathrm{A}, \triangle: \mathrm{D}, \square: \mathrm{G}, \diamond: \mathrm{J}$. Bars on each point $(n=3)$ are standard deviations of triplicate measurement.

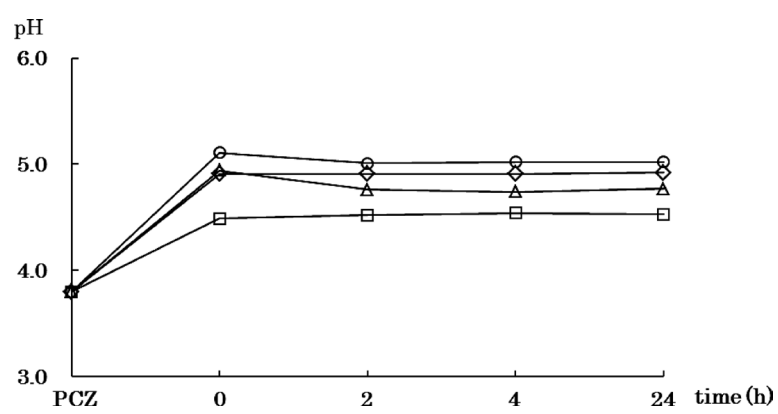

Fig. 3. Time Course of Changes in $\mathrm{pH}$ over Time after Mixing PCZ Oral Solution with Tea-Based Drinks (A, D, G and J)

$\mathrm{O}: \mathrm{A}, \triangle: \mathrm{D}, \square: \mathrm{G}, \diamond: \mathrm{J}$.

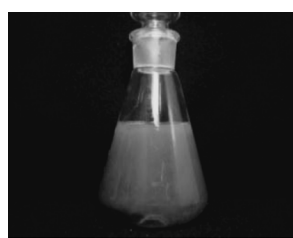

just after mixing

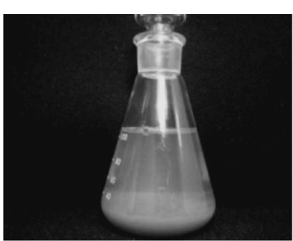

after 2 hours

Content (\%)

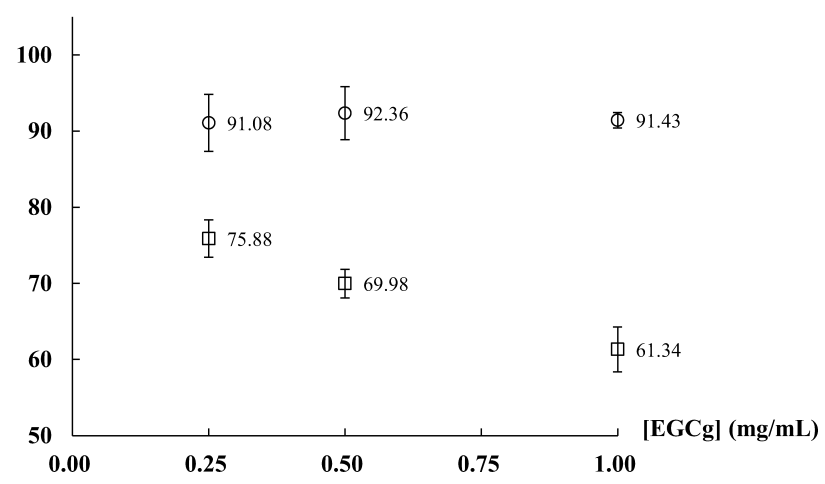

Fig. 5. The Content (\%) of PCZ and EGCg vs. the Concentration of EGCg Solution Mixed with the PCZ Oral Solution

$\square$ : PCZ, O: EGCg. Bars on each point $(n=3)$ are standard deviations of triplicate measurement.

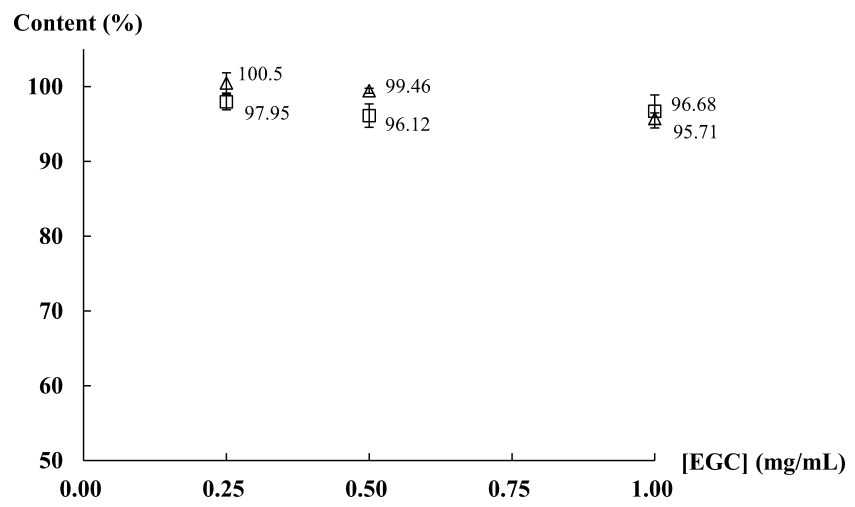

Fig. 6. The Content (\%) of PCZ and EGC vs. the Concentration of EGC Solution Mixed with the PCZ Oral Solution

$\square$ : PCZ, $\triangle$ : EGC. Bars on each point $(n=3)$ are standard deviations of triplicate measurement.

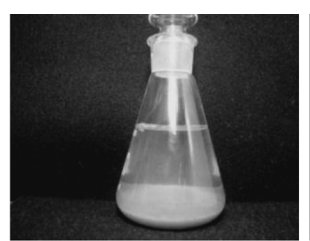

after 4 hours

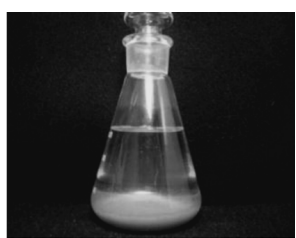

after 24 hours

Fig. 4. Time Course of Changes in Turbidity over Time after Mixing of PCZ Oral Solution and Tea-Based Drink G

insoluble substance was gradually precipitated in the solution. Figure 4 shows the time course of changes in turbidity of the mixture of the PCZ oral solution and G.

It was speculated that the changes in $\mathrm{pH}$ and turbidity of the mixture were related to the decreased PCZ content shown in Figs. 1 and 2. PCZ is a weak base; therefore, the increase in the $\mathrm{pH}$ of the mixture might have led to a decrease in the solubility of PCZ, thus decreasing the PCZ content.

Changes in PCZ Content after Mixing the PCZ Oral Solution with the Green Tea Polyphenol Solution We then performed a pilot study to elucidate the basis for decreased PCZ content after mixing the PCZ oral solution with tea-based drinks. According to the experimental results of mixing the PCZ oral solution with green tea drink (A, B, and C) as shown in Table 2, it is thought that one of the factors controlling the changes in PCZ content is green tea polyphenol. The EGCg and EGC were used for this study, because approximately $70-80 \%$ of polyphenol in green tea are EGCg and EGC. 9) The PCZ oral solution was mixed with the green tea polyphenol (EGCg or EGC) solution in order to calculate the residual PCZ and polyphenol content. The polyphenol solution was prepared using a citrate buffer solution ( $\mathrm{pH}$ 5.2) to reproduce the increased $\mathrm{pH}$ after mixing. Figures 5 and 6 show the results. The $\mathrm{pH}$ of all solutions after mixing was 5.2.

The mixture of the PCZ oral solution and the EGCg solution became cloudy immediately after mixing. In contrast, the mixture containing PCZ and EGC did not become cloudy. As shown in Fig. 5, the residual PCZ content decreased 
considerably with an increase in the concentration of EGCg solution. Unlike the case of PCZ, the residual EGCg content was approximately $91-92 \%$ regardless the concentration of EGCg solution. When the PCZ oral solution was mixed with the EGC solution, the residual PCZ and EGC content hardly declined as shown in Fig. 6. The main factor of the content decline of PCZ by mixing PCZ oral solution with green tea drink was proved to be the polyphenol with galloyl group such as EGCg. Therefore, it is thought that the polyphenol with a galloyl group included in tea-based drink causes a content decline of PCZ.

In addition, the $\mathrm{pH}$ of the mixture $(0.50 \mathrm{~mL}$ of the $\mathrm{PCZ}$ oral solution was mixed with $16.5 \mathrm{~mL}$ of the citrate buffer solution (pH 5.2)) in the control experiment increased to 5.2, but the residual PCZ content did not decrease. This result indicated that the reduction in PCZ content after mixing the PCZ oral solution with the tea-based drinks was not caused by decreased solubility of PCZ at a higher $\mathrm{pH}$.

The results obtained in this pilot study demonstrated that PCZ content was reduced after dilution in tea-based drinks because of the interaction between PCZ and the polyphenol with a galloyl group in tea-based drinks.

\section{Conclusion}

In most cases, the mixture of the $\mathrm{PCZ}$ oral solution with tea-based drinks caused the precipitation of insoluble substances and a reduction in the residual PCZ content. This could result in an insufficient therapeutic effect for patients with schizophrenia and requires special caution.

Especially, it was found that the residual PCZ content in the mixture decreased with an increase in the content of the green tea polyphenol. This study demonstrated that PCZ's incompatibility with tea-based drinks was caused by polyphenol with a galloyl group such as EGCg.

In the future, we plan to perform NMR, MS, thermal analysis, and quantum chemistry calculations to elucidate the mechanism of interaction between PCZ and the polyphenol in the tea-based drinks.

\section{References}

1) Keith S. J., Kane J. M., J. Clin. Psychiatry, 64, 1308-1315 (2003).

2) Kamijima K., Owashi T., Takahashi T., Nakagome K., Psychiatria et Neurologia Japonica, 107, 696-703 (2005).

3) Package insert of Neuleptil ${ }^{\circledR}$ issued by Shionogi \& Co., Ltd., Osaka, Japan.

4) Ikeda H., Moriwaki H., Yukawa M., Iwase Y., Aki H., Yakugaku Zasshi, 130, 1589-1595 (2010).

5) Ikeda H., Moriwaki H., Matsubara T., Yukawa M., Iwase Y., Yukawa E., Aki H., Yakugaku Zasshi, 132, 145-153 (2012).

6) Yamaguchi N., Sakai T., Miyamoto S., Yoshio T., Morokawa Y., "Kokoro No Chiryou-Yaku Hand Book," 6th ed., Seiwa Shoten Co., Ltd., Tokyo, 2010, p. 138.

7) Yoshida M., Japanese Pharmacology \& Therapeutics, 10, 579-589 (1982).

8) Aki H., Ohta M., Fukusumi K., Okamoto Y., Jpn. J. Pharm. Health Care Sci., 32, 190-198 (2006).

9) Isemura M., "Health Beneficial Functions and Development of Tea Goods," CMC Publishing Co., Ltd., Tokyo, 2006, p. 16. 